\title{
Neat1 regulates oxidized low-density lipoprotein-induced inflammation and lipid uptake in macrophages via paraspeckle formation
}

\author{
NING HUANG-FU, JING-SONG CHENG, YONG WANG, ZHEN-WEI LI and SHENG-HUANG WANG \\ Department of Cardiology, Ningbo First Hospital, Ningbo, Zhejiang 315010, P.R. China
}

Received August 18, 2017; Accepted November 28, 2017

DOI: $10.3892 / \mathrm{mmr} .2017 .8211$

\begin{abstract}
Oxidized low-density lipoprotein (oxLDL) induces macrophage inflammation and lipid uptake, and serves important roles in the development of atherosclerosis. The long non-coding RNA (lncRNA) nuclear paraspeckle assembly transcript 1 (neat1) has two isoforms; the longer isoform, neat1_2, mediates the formation of subnuclear structures called paraspeckles. Reverse transcription-quantitative polymerase chain reaction (RT-qPCR), western blotting and RNA protein immunoprecipitation (RIP), revealed that oxLDL induced paraspeckle formation in the THP-1 cell line. Additionally, the nuclear factor- $\kappa \mathrm{B}$ and $\mathrm{p} 38$ pathways were observed to be involved in neat1 transcription. To investigate the role of paraspeckles in oxLDL-induced macrophage inflammation and lipid uptake, macrophages were transfected with small interfering RNAs against NEAT1, NEAT1_2, non-POU domain-containing octamer-binding (NONO) and splicing factor proline and glutamine rich prior to oxLDL incubation. In addition, inflammation-associated pathways and scavenger receptors were analyzed by performing western blotting and RT-qPCR. p65 phosphorylation and cluster of differentiation 36 (CD36) were demonstrated to serve roles in paraspeckle-mediated inflammation and lipid uptake, respectively. To determine the underlying mechanism, RIP was preformed, which revealed that NONO binds CD36 mRNA to decrease its expression. In conclusion, oxLDL induced neat1_2-mediated paraspeckle formation. Paraspeckles participate in oxLDL-induced macrophage inflammation and lipid uptake by regulating p65 phosphorylation and CD36 mRNA.
\end{abstract}

Correspondence to: Dr Sheng-Huang Wang, Department of Cardiology, Ningbo First Hospital, 59 Liuting Road, Ningbo, Zhejiang 315010, P.R. China

E-mail: 13605746913@163.com

Key words: oxidized low-density lipoprotein, NEAT1, inflammation, lipid uptake, paraspeckle

\section{Introduction}

Atherosclerosis is an inflammatory disease about creating an atheromatous plaque (1-3). The pathobiology of atherosclerotic lesions is very complicated, but generally, macrophages-derived foam cells contributes to rupture of unstable plaques (4). Ruptures of the fibrous cap expose thrombogenic material, eventually induce thrombus formation in the lumen, resulting in ischemia (5). Research into the disease has led to many compelling hypotheses about the pathophysiology of atherosclerotic lesion formation and of complications such as myocardial infarction and stroke (6).

Macrophages engulf modified lipoproteins and transform themselves into lipid-loaded foam cells, which contribute to the formation of the necrotic core in atheromatous plaques (7). Various inflammatory factors secreted by lipid-loaded macrophages also enlarge and expand the local inflammatory reaction, which aggravates atherosclerosis (6). The investigation of the mechanism involved in oxidized low-density lipoprotein (oxLDL)-induced macrophage inflammation and lipid uptake is becoming increasingly important.

Long non-coding RNAs (lncRNAs) are non-coding RNAs that are longer than $200 \mathrm{bp}$. The lncRNA nuclear paraspeckle assembly transcript 1 (NEAT1) is widely expressed in various tissues and participates in many biological activities, such as adipogenesis and tumorigenesis, including breast cancer, leukemia, ovarian cancer, hepatocellular carcinoma and laryngeal squamous cancer (8-13). In a recent study, neat1_2, a longer isoform of neat1, together with RNA-binding proteins, including PSPC1, non-POU domain-containing octamer-binding (NONO) and SFPQ, initiated the formation of subnuclear structures called paraspeckles (14). Paraspeckles can exert antiviral functions by stabilizing SFPQ, which suppresses IL8 transcription (15).

In this study, we explored the role of neat1-mediated paraspeckle formation in oxLDL-induced macrophage inflammation and lipid uptake.

\section{Materials and methods}

Cell culture and transfection. The human monocyte cell line THP-1 was purchased from American Type Culture Collection (ATCC; Manassas, VA, USA). The cells were cultured in complete medium consisting of $10 \%$ fetal bovine serum and 
RIPM-1640 (both from Gibco, Grand Island, NY, USA) with penicillin and streptomycin (Sigma-Aldrich; Merck KGaA, Darmstadt, Germany) at $37^{\circ} \mathrm{C}$ with $5 \% \mathrm{CO}_{2}$. Before treatment, the cells were supplied with $100 \mathrm{ng} / \mathrm{ml}$ PMA (79346; Sigma-Aldrich; Merck KGaA) to differentiate them into macrophages. Inhibitors including SB20358 (p-p38 inhibitor, S8307), SP600125 (p-JNK inhibitor, S5567), PD98059 (p-ERK inhibitor, P215) and BAY11-7085 (p-p65 inhibitor, B5681) (all purchased from Sigma-Aldrich; Merck KGaA), were added $30 \mathrm{~min}$ prior to oxLDL ( $40 \mu \mathrm{g} / \mathrm{ml}$, YB-002; Yiyuan Biotechnology Co., Ltd., Guangzhou, China) treatment for 24 h. Dimethyl sulphoxide (DMSO, D2650; Sigma-Aldrich) was used as the control of inhibitors since these chemical inhibitors were dissolved in DMSO. Finally, the cells were harvested for later experiments.

siRNAs against NEAT1_1 (GGAACAUUCUCAUUU AAUAtt), NEAT1_2 (GGGUAAAUCUCAAUCUUAAtt), NONO (GGGGUGGUAUUAAACAAGUCA) and SFPQ (GGCAAAGGAUUCGGAUUUAUU), as well as a negative control (nc) (GUACCUGACUAGUCGCAGAAG) were synthetized by Ruibo Biotechnology Co., Ltd. (Guangzhou, China). Transfection was performed using Lipo 3000 reagent (Life Technologies, Grand Island, NY, USA) according to the manufacturer's instructions. After transfection with siRNAs $(2 \mu 1,50 \mathrm{nM})$ and culturing for $24 \mathrm{~h}$, the cells were supplied with human oxLDL (40 $\mu \mathrm{g} / \mathrm{ml}$, YB-002; Yiyuan Biotechnology) for the indicated lengths of time.

RNA isolation and reverse transcription-quantitative polymerase chain reaction ( $R T-q P C R)$. Total RNA was extracted using a simple total RNA extraction kit (Tiangen, Beijing, China). cDNA was synthesized using random primers (Takara, Otsu, Japan). SYBR premix Ex Taq II (Takara) master mix was used for RT-qPCR analysis, and the amplification consisted of 95 for $30 \mathrm{sec}$ and 40 cycles of 95 for $5 \mathrm{sec}$ and 60 for $30 \mathrm{sec}$. RNA $(18 \mathrm{sec})$ served as an endogenous control. Primers were as follows: NEAT1 forward, GAGAACCAAAGGGAGGGG TG and reverse, TGCTGCGTATGCAAGTCTGA; NEAT1_2 forward, ACATTGTACACAGCGAGGCA and reverse, CAT TTGCCTTTGGGGTCAGC; $\beta$-actin forward, TGACGTGGA CATCCGCAAAG and reverse, CTGGAAGGTGGACAG CGAGG.

Western blot analysis. The cells were harvested and lysed using total protein lysis buffer (Cell Signaling Technology, Inc., Danvers, MA, USA). A total of $30 \mu \mathrm{g}$ of protein was separated on a $10 \%$ polyacrylamide-SDS gel, blotted onto a PVDF membrane (Millipore, Billerica, MA, USA) and blocked with 5\% non-fat milk (Sangon Biotech Co., Ltd., Shanghai, China). After incubation with the primary antibody overnight at $4^{\circ} \mathrm{C}$ and the corresponding secondary antibody at $37^{\circ} \mathrm{C}$ for $1 \mathrm{~h}$, the membrane was developed using an ECL kit (Pierce, Rockford, IL, USA). The antibodies used were as follows: Rabbit anti-GAPDH (1:1,000) (GP10353; Nuoyang, Hangzhou, China); rabbit anti-p-p65 (1:1,000; no. 3033); rabbit anti-p-p38 (1:1,000; no. 4511); rabbit anti-p-JNK (1:1,000; no. 9255); rabbit anti-p-ERK (1:1,000; no. 3510) (all from Cell Signaling Technology, Inc.); rabbit anti-cluster of differentiation 36 (CD36; 1:1,000; ab133625); rabbit anti-LOX-1 (1:1,000; ab60178); rabbit anti-NONO (1:1,000; ab70335); rabbit
anti-SFPQ (1:1,000; ab38148) (all from Abcam, Shanghai, China); goat anti-rabbit (1:5,000; GP853); and goat anti-mouse $(1: 5,000 ;$ GP843) (both from Nuoyang).

Nuclear protein isolation. Nuclear protein was extracted using a Nuclear and Cytoplasmic Protein Extraction kit (Beyotime Institute of Biotechnology (Shanghai, China) according to the manufacturer's instructions. For nuclear RNA extraction, after centrifugation to isolate the cytoplasmic proteins, the pellet was dissolved using TRIzol reagent (Life Technologies), then extracted using chloroform and precipitated using isopropanol.

RIP. RNA protein immunoprecipitation was performed using a Magna RIP kit (Millipore). In brief, the cells were washed with ice-cold PBS and lysed on ice with RIP lysis buffer. A/G magnetic beads with antibodies against IgG (Millipore), NONO or SFPQ (Abcam, Cambridge, MA, USA) were allowed to settle for $30 \mathrm{~min}$ at room temperature. Then, the cell lysate was immunoprecipitated with the antibody-coated magnetic beads. After incubating the magnetic-bead-bound complexes with $10 \%$ SDS and proteinase $\mathrm{K}$, the supernatant was used for RNA extraction. The first-strand cDNA was synthesized using a cDNA synthesis kit (Applied Biosystems, Foster City, CA, USA). Finally, qRT-PCR was performed for further analysis.

Oil red staining. After incubating with oxLDL $(40 \mu \mathrm{g} / \mathrm{ml})$ for $24 \mathrm{~h}$, the cells were washed with PBS and stained using oil red stain solution, which comprised $30 \%$ alcohol and $70 \%$ oil red solution. Then, the cells were further stained with hematoxylin solution for $10 \mathrm{~min}$. After washing with PBS, the cells were observed using an Olympus light microscope (Olympus, Tokyo, Japan). Oil red solution and hematoxylin were purchased from Jiancheng Bioengineering Institute (Nanjing, China).

Statistical analysis. Data are shown as the mean \pm standard deviation (SD). Non parametric t-tests were used to compare the differences between two groups. One-way analysis of variance (ANOVA) was used to compare the differences among three or more groups. P-values $<0.05$ were considered to indicate a statistically significant difference.

\section{Results}

OxLDL induces NEAT1 and NEAT1_2 expression via 38 and $N F-\kappa B$ signaling. The lncRNA NEAT1 has two transcripts, NEAT1_1 and NEAT1_2, which have the same transcription initiation site. Because the entire NEAT1_1 sequence overlapped with the 5' sequence of NEAT1_2, we designed two primer pairs, NEAT1, to detect the expression of both NEAT1_1 and NEAT1_2, and NEAT1_2, to detect only NEAT1_2 (Fig. 1A). When the macrophages were incubated with human oxLDL ( $30 \mu \mathrm{g} / \mathrm{ml})$ for $2,4,6,8$ or $10 \mathrm{~h}$, we found that both neat1 and NEAT1_2 increased over time (Fig. 1B). Next, the macrophages were stimulated with different concentrations of oxLDL (from 10 to $50 \mu \mathrm{g} / \mathrm{ml}$ ) for $24 \mathrm{~h}$. As shown in Fig. 1C, NEAT1 increased, but NEAT1_2 showed no significant changes, which means that half-life period of NEAT1_2 is shorter than NEAT1_1. To identify the regulatory pathway involved, MAPK and NF- $\mathrm{KB}$ inhibitors were used. We found 
A

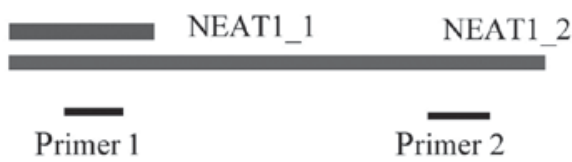

B

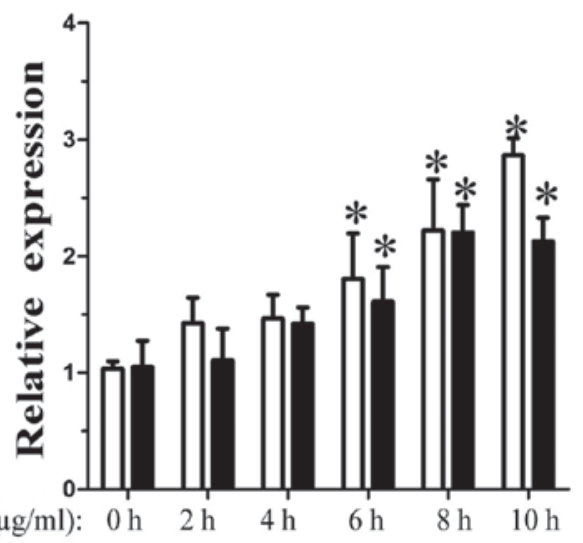

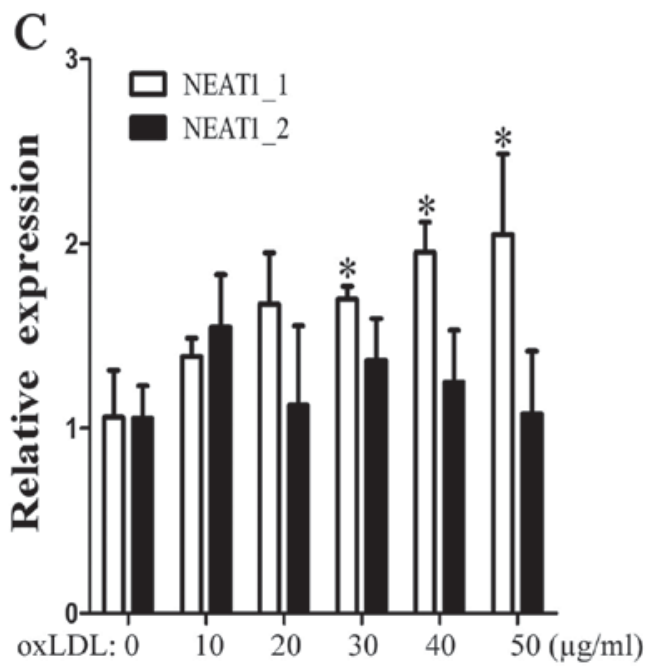

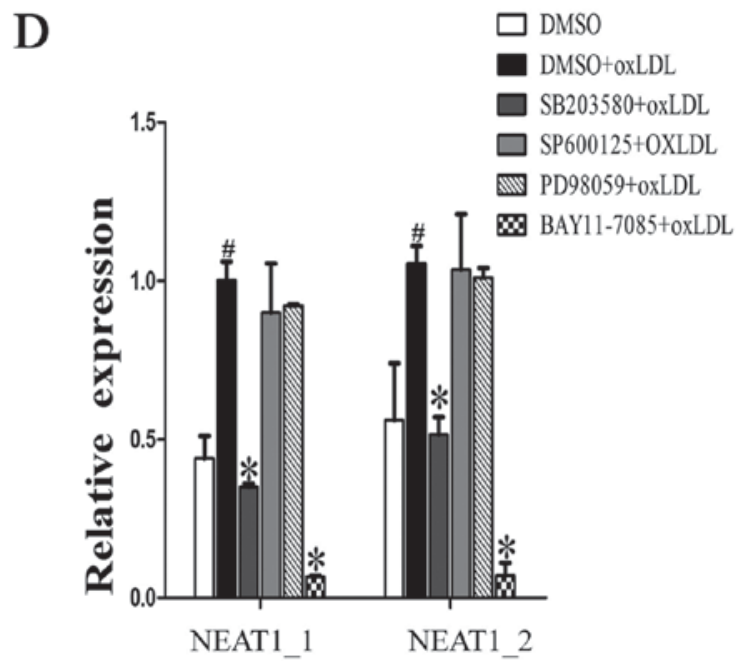

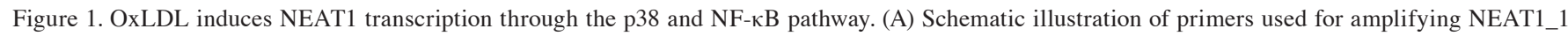
and NEAT1_2. Primer 1 was used to detect the NEAT1_1 and NEAT1_2 transcripts. Primer 2 was used to specifically detect NEAT1_2. (B) Macrophages were treated with $30 \mu \mathrm{g} / \mathrm{ml}$ oxLDL for the indicated times (from 2 to $10 \mathrm{~h}$ ). RT-qPCR was performed to detect the expression of NEAT1_1 and NEAT1_2. ${ }^{*} \mathrm{P}<0.05$ vs. 0 h. (C) Macrophages were treated with different concentrations of oxLDL (from 10 to $50 \mu \mathrm{g} / \mathrm{ml}$ ) for $24 \mathrm{~h}$. RT-qPCR was performed to detect the expression of NEAT1_1 and NEAT1_2. "P<0.05 vs. $0 \mu \mathrm{g} / \mathrm{ml}$. (D) Prior to oxLDL treatment, macrophages were incubated with DMSO or different chemical inhibitors (SB203580, a p38 inhibitor; SP600125, a c-Jun N-terminal kinase inhibitor; PD98059, an extracellular signal-regulated kinases 1/2 inhibitor; PDTC, an nuclear factor- $\kappa$ B inhibitor) for $30 \mathrm{~min}$. RT-qPCR was performed to detect the expression of NEAT1_1 and NEAT1_2. ${ }^{\mathrm{P}}<0.05$ vs. DMSO; ${ }^{*} \mathrm{P}<0.05$ vs. DMSO + oxLDL. oxLDL, oxidized low-density lipoprotein; NEAT, nuclear paraspeckle assembly transcript 1 ; RT-qPCR, reverse transcription-quantitative polymerase chain reaction.

that p38 and NF- $\mathrm{BB}$ mediated oxLDL-induced NEAT1 and NEAT1_2 expression (Fig. 1D). The above results show that oxLDL can induce neat1 and NEAT1_2 transcription and that NEAT1_2 was less stable than neat1_1.

OxLDL induces NEAT1_2-mediated paraspeckle formation. In previous study, the continued transcription of the IncRNA NEAT1_2 promotes the formation of subnuclear structures called paraspeckles. First, we detected the expression of two paraspeckle-related proteins, NONO and SFPQ, during oxLDL incubation. As shown in Fig. 2B and C, SFPQ did not significantly change over the indicated times or with the indicated concentrations of oxLDL. However, under the same conditions, oxLDL can promote nono expression. To detect whether oxLDL can induce paraspeckle formation, RIP experiments were used to detect cross-linking between nono and NEAT1 or NEAT1_2. As shown in Fig. 2A, after $10 \mathrm{~h}$ of oxLDL stimulation, the complex that was immunoprecipitated using a NONO antibody contained NEAT1_2 but not neat1. Our results show that oxLDL stimulation can induce NEAT1_2-mediated paraspeckle formation.

Neat1 promotes TNF- $\alpha$ secretion by inducing p65 phosphorylation. To investigate whether paraspeckles participate in the oxLDL-mediated secretion of proinflammatory factors, macrophages were transfected with siRNAs against NEAT1_1, NEAT1_2, NONO or SFPQ for 24 h prior to oxLDL stimulation. As shown in Fig. 3A, TNF- $\alpha$ and iNOS transcription were reduced by transfection with either siR-NEAT1_1 or siR-NEAT1_2. Then, we measured iNOS protein expression, but we found no significant changes after siRNA transfection (Fig. 3B). TNF- $\alpha$ expression in the supernatant of macrophages transfected with siRNAs under oxLDL treatment was also analyzed, and we found that knocking down NEAT1_1 or NEAT1_2 indeed decreased oxLDL-induced TNF- $\alpha$ secretion (Fig. $3 \mathrm{C}$ ). To determine whether neat1 regulates TNF- $\alpha$ secretion by affecting proinflammatory pathways, 
A

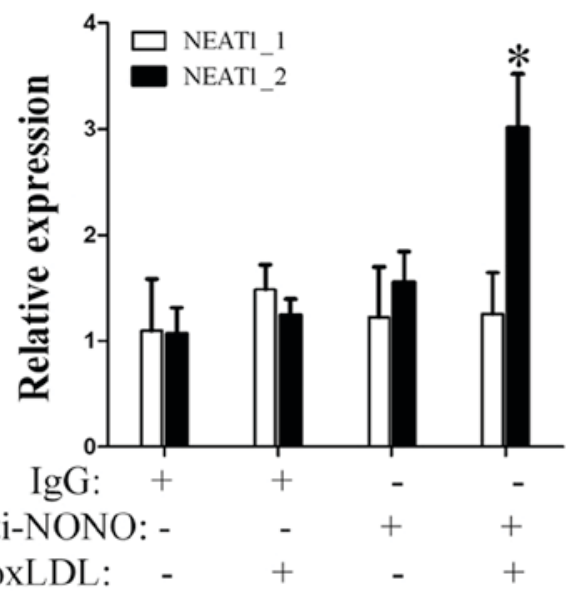

B

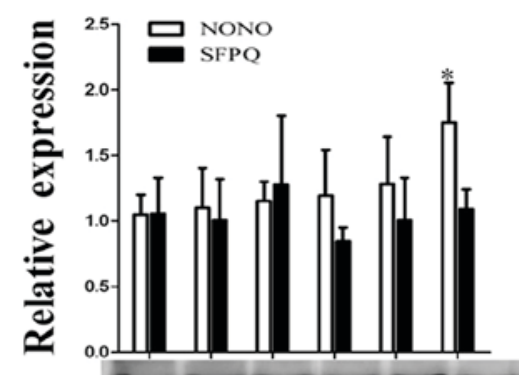

GAPDH $37 \mathrm{kDa}$

$\mathrm{SFPQ} \longrightarrow 95 \mathrm{kDa}$

$\mathrm{NONO}=-\longrightarrow 60 \mathrm{kDa}$

$\mathrm{C}$

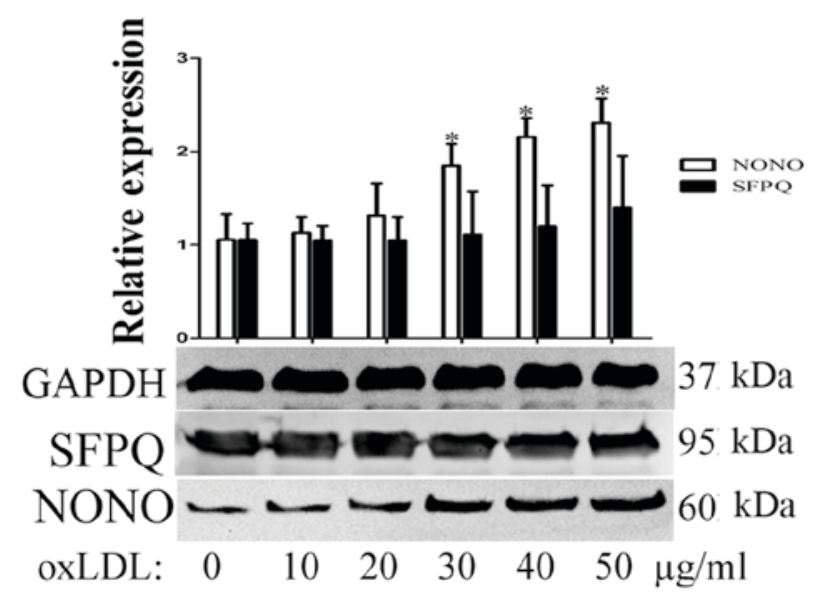

Figure 2. OxLDL promotes NEAT1_2-mediated paraspeckle formation. (A) Macrophages were treated with oxLDL for 8 h. RNA protein immunoprecipitation was performed using anti-NONO (with IgG as control), and reverse transcription-quantitative polymerase chain reaction was performed to detect the expression of NEAT1_1 and NEAT1_2 in the immunoprecipitated complex. * $\left.\mathrm{P}<0.05 \mathrm{vs.} \mathrm{NEAT1 \_ 1.} \mathrm{(B)} \mathrm{Macrophages} \mathrm{were} \mathrm{treated} \mathrm{with} \mathrm{oxLDL} \mathrm{(30} \mu \mathrm{g} / \mathrm{ml}\right)$ for the indicated times. NONO and SFPQ were detected by western blotting, with GAPDH as an endogenous control. *P<0.05 vs. 0 h. (C) Macrophages were treated with different concentrations of oxLDL (from 10 to $50 \mu \mathrm{g} / \mathrm{ml}$ ) for $24 \mathrm{~h}$. NONO and SFPQ were detected by western blotting, with GAPDH as an endogenous control. "P<0.05 vs. 0 g/ml. oxLDL, oxidized low-density lipoprotein; NEAT1, nuclear paraspeckle assembly transcript 1; IgG, immunoglobulin G; NONO, non-POU domain-containing octamer-binding; SFPQ, splicing factor proline and glutamine rich.

we analyzed the expression of MAPKs and NF- $\mathrm{BB}$. As shown in Fig. 3D and E, NEAT1 knockdown had no significant effect on MAPKs or NF- $\mathrm{KB}$ at the early stage of activation $(2 \mathrm{~h})$ by oxLDL, but it did decrease ERK and p65 phosphorylation at a later stage $(8 \mathrm{~h})$. The above results show that NEAT1 promotes oxLDL-induced TNF- $\alpha$ secretion by regulating MAPKs and $\mathrm{NF}-\kappa \mathrm{B}$.

Neat1 suppresses lipid uptake by binding CD36 mRNA. Since lipid uptake by macrophages plays an important role in the development of atherosclerosis, we also explored the effect of paraspeckles on lipid uptake in macrophages. As shown in Fig. 4A, transfection with either siR-NEAT1_1 or siR-NEAT1_2 promotes Dil-labeled oxLDL (Dil-oxLDL) uptake by macrophages. To determine the mechanism, we also analyzed the transcription of scavenger receptors, including SRA, CD36 and LOX-1, after transfection with siR-NEAT1_1 or siR-NEAT1_2 under oxLDL treatment. As shown in Fig. 4B, only transfection with siR-NEAT1_2 promotes CD36 and LOX-1 transcription. However, we found that transfection with either siR-NEAT1_1 or siR-NEAT1_2 promotes CD36 protein expression (Fig. 4C). We suspect that NEAT1_2 inhibits CD36 expression by stabilizing CD36 mRNA in paraspeckles. R-IPs were performed using the anti-NONO antibody. When macrophages were treated with oxLDL, the immunoprecipitated complex contained more CD36 mRNA (Fig. 4D). In conclusion, NEAT1 suppressed lipid uptake in part by stabilizing CD36 mRNA in paraspeckles.

\section{Discussion}

Inflammation and lipid uptake in macrophages by oxLDL play pivotal roles in the formation of atherosclerotic plaques during the development of atherosclerosis. A deeper understanding of the mechanism involved in the oxLDL-induced secretion of proinflammatory factors and uptake of lipids by macrophages may yield therapeutic targets for atherosclerosis. In this study, we explore the possibility of lncRNAs participating in oxLDL-induced secretion of proinflammatory factors and lipid uptake by macrophages. Because 
A

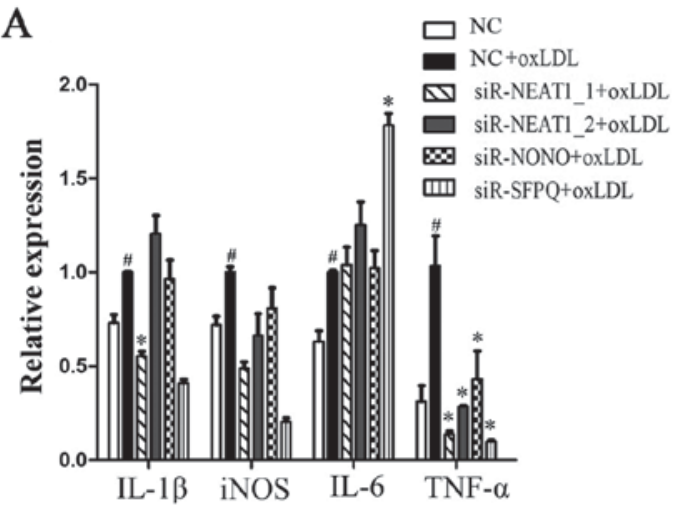

$\mathrm{C}$

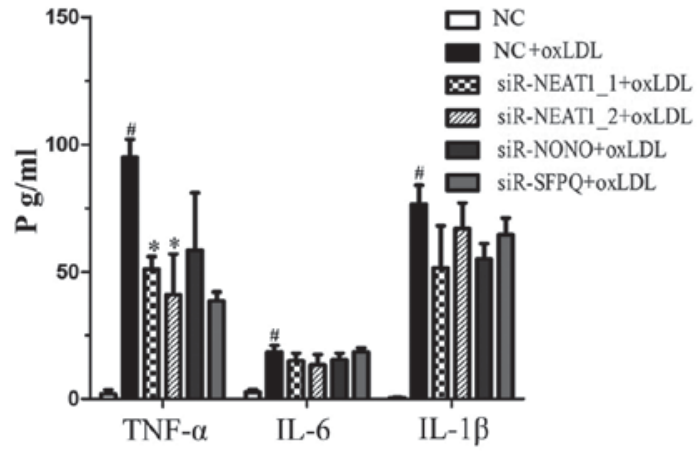

E

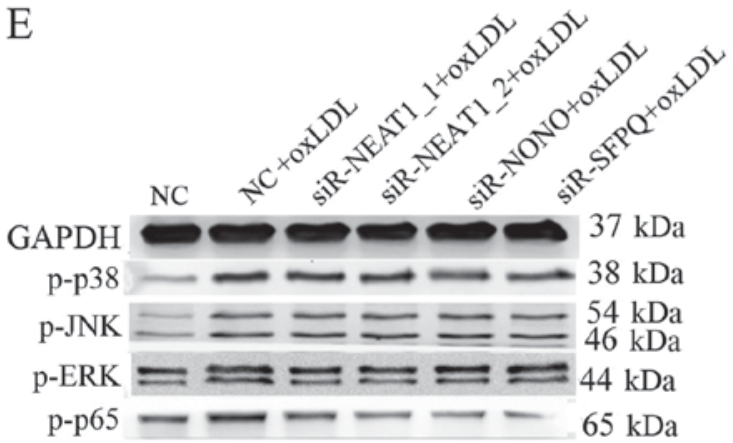

B

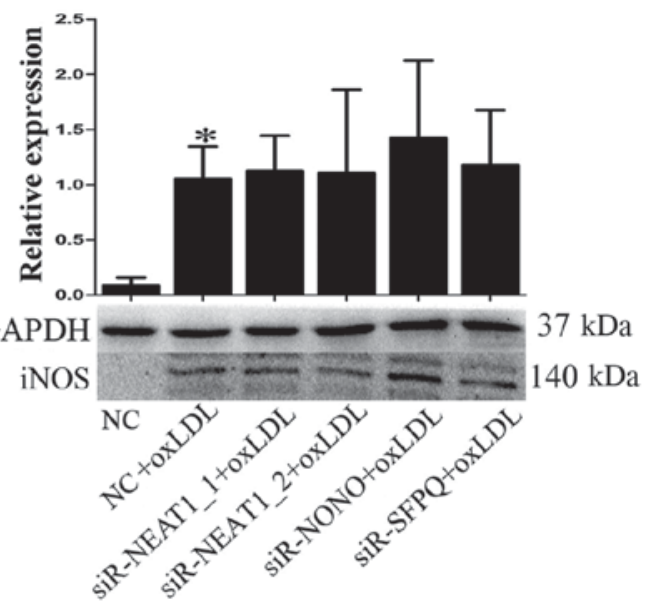

$\mathrm{D}$

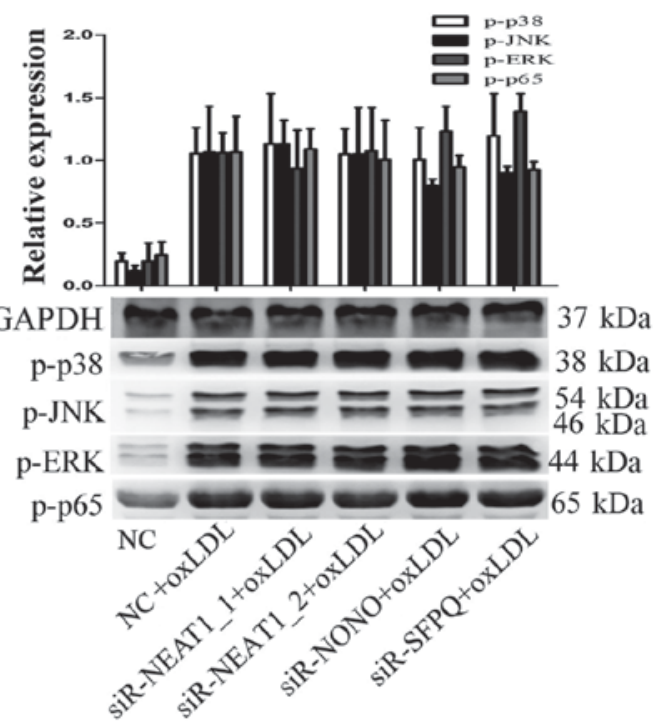

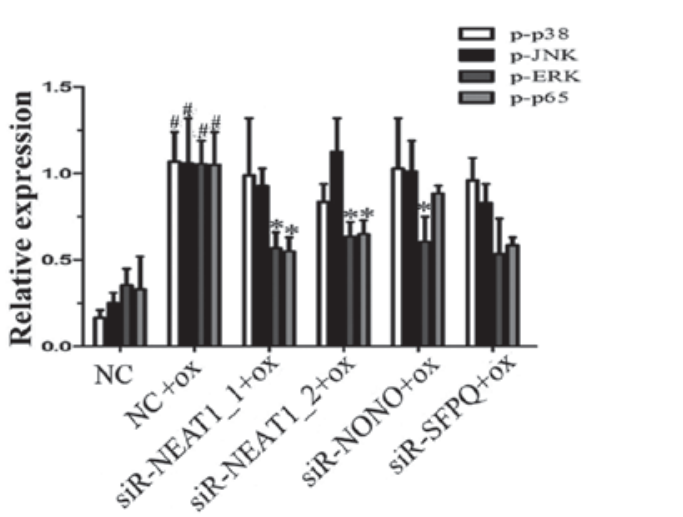

Figure 3. NEAT1 promotes TNF- $\alpha$ secretion by activating p65 phosphorylation. Prior to oxLDL treatment, macrophages were transfected with a negative control, siR-NEAT1_1, siR-NEAT1_2, siR-NONO or siR-SFPQ for $24 \mathrm{~h}$. (A) Reverse transcription-quantitative polymerase chain reaction was performed to detect gene transcription. (B) Following transfection, macrophages were incubated with oxLDL for a further $24 \mathrm{~h}$. Western blotting was performed to detect iNOS protein expression, and GAPDH was an endogenous control. (C) Following transfection macrophages were incubated with oxLDL for a further $10 \mathrm{~h}$. The supernatant was collected to analyze the levels of secreted TNF- $\alpha$, IL-6 and IL-1B. (D) Following transfection macrophages were incubated with oxLDL for $2 \mathrm{~h}$. Western blotting was performed to detect p-p38, p-JNK, p-ERK and p-p65 protein expression, and GAPDH was an endogenous control. (E) Following transfection macrophages were incubated with oxLDL for a further $8 \mathrm{~h}$. Western blotting was performed to detect p-p38, p-JNK, p-ERK and p-p65 protein expression, and GAPDH was an endogenous control. " $\mathrm{P}<0.05$ vs. NC; "P $<0.05$ vs. NC + oxLDL. oxLDL, oxidized low-density lipoprotein; TNF- $\alpha$, tumor necrosis factor- $\alpha$; NEAT, nuclear paraspeckle assembly transcript 1 ; NONO, non-POU domain-containing octamer-binding; SFPQ, splicing factor proline and glutamine rich; IL, interleukin; p, phosphorylated; JNK, c-Jun N terminal kinase; ERK, extracellular signal-regulated kinase; siR, small interfering RNA.

NEAT1_2 may contribute to the formation of subnuclear structures called paraspeckles (16), we mainly investigate the role of paraspeckles in inflammation and lipid uptake. By transfecting macrophages with siRNAs against NEAT1, 
A

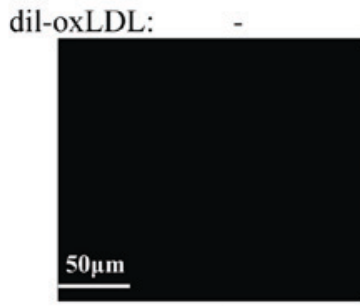

$\mathrm{NC}$

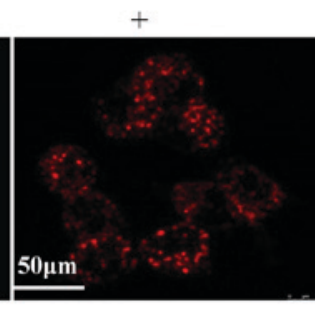

$\mathrm{NC}$

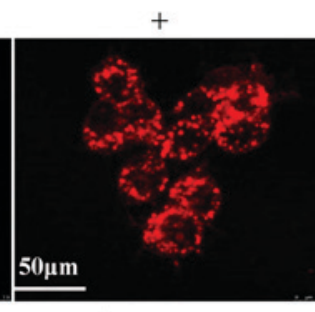

siR-NEAT1_1
$+$

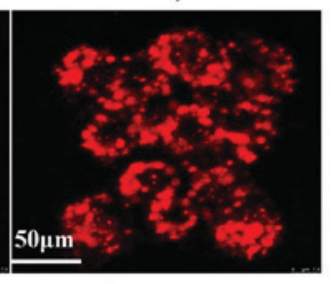

siR-NEAT1_2

$\mathrm{B}$

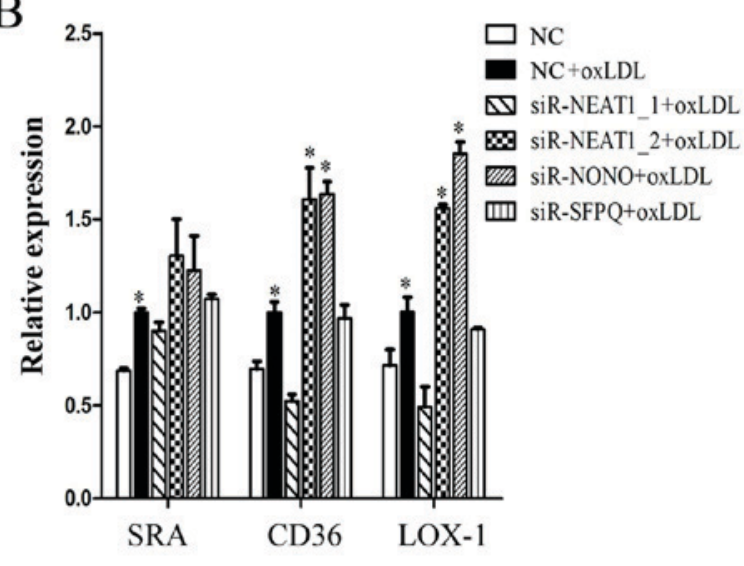

C

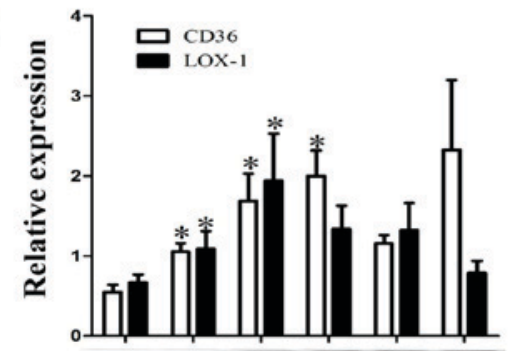

GAPDH

CD36

LOX-1

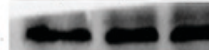

36

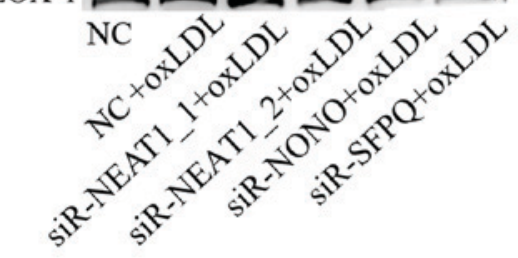

D

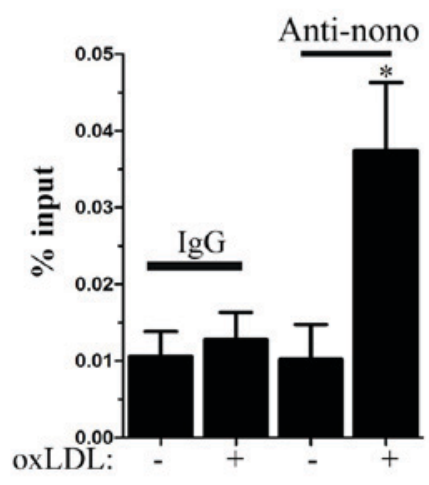

Figure 4. NEAT1 inhibits lipid uptake in part by suppressing CD36 expression. Macrophages were transfected with a negative control, siR-NEAT1_1 or siR-NEAT1_2 for $24 \mathrm{~h}$ and (A) then incubated with Dil-oxLDL for $30 \mathrm{~min}$. Lipid uptake was evaluated based on the fluorescence intensity of Dil-oxLDL. Scale bars, $50 \mu \mathrm{m}$. (B) Macrophages were transfected and then incubated with oxLDL for $12 \mathrm{~h}$. RT-qPCR was performed to detect the transcription of associated genes. (C) Macrophages were transfected and then incubated with oxLDL for $24 \mathrm{~h}$. Western blotting was performed to detect CD36 and LOX-1 protein expression, and GAPDH was an endogenous control. "P<0.05 vs. NC + oxLDL. (D) Macrophages were treated with oxLDL for $8 \mathrm{~h}$. RNA protein immunoprecipitation was performed using anti-nono (with IgG as control), and RT-qPCRs were used to detect the level of CD36 mRNA in the immunoprecipitated complex. ${ }^{*} \mathrm{P}<0.05$ vs. (-) oxLDL. NEAT, nuclear paraspeckle assembly transcript 1; CD36, cluster of differentiation 36; siRNA, small interfering RNA; oxLDL, oxidized low-density lipoprotein; Dil-oxLDL, Dil-labeled oxLDL; RT-qPCR, reverse transcription-quantitative polymerase chain reaction; LOX-1, lectin-like oxidized low-density lipoprotein receptor 1; SRA, serum resistance associated protein; NONO, non-POU domain-containing octamer-binding; SFPQ, splicing factor proline and glutamine rich; IgG, immunoglobulin G.

NEAT1_2, NONO or SFPQ, which would stop paraspeckle formation (17), we found that paraspeckles have different functions in inflammation and lipid uptake: Paraspeckles promote TNF- $\alpha$ secretion indirectly and inhibit CD36 expression by directly binding CD36 mRNA. Moreover, we also found that the constitutive paraspeckle protein NONO may perform its function independently of paraspeckles (Fig. 3E). But in our present study, the detailed mechanism by which paraspeckles regulate p65 phosphorylation or paraspeckles pesist CD36 mRNA is unknown. The role of paraspekcle in other biological activities of macrophages such as migration, chemotaxis and apoptosis is also needed to be explored. Collectively, these results illustrate the complexity of paraspeckles: Paraspeckles may affect biological activities by stabilizing target mRNAs or proteins, and these stabilized proteins may function as transcription factors to activate the transcription of other genes (15). In our future research, we aim to explore the detailed mechanism by which paraspeckles regulate p65 phosphorylation. Additionally, we want to understand whether paraspeckles influence TNF- $\alpha$ transcription by directly changing the activity of the promoter.

In conclusion, we first explored the function of NEAT1- and NEAT1_2-mediated paraspeckle formation in oxLDL-induced secretion of proinflammatory factors and lipid uptake by macrophages. Paraspeckles promote TNF- $\alpha$ secretion partially by regulating p65 phosphorylation and suppressed lipid uptake partially by stabilizing CD36 mRNA, which decreases CD36 expression. 


\section{References}

1. Ross R: The pathogenesis of atherosclerosis: A perspective for the 1990s. Nature 362: 801-809, 1993.

2. Hansson GK and Hermansson A: The immune system in atherosclerosis. Nat Immunol 12: 204-212, 2011.

3. Ross R: Atherosclerosis-an inflammatory disease. N Engl J Med 340: 115-126, 1999.

4. Finn AV, Nakano M, Narula J, Kolodgie FD and Virmani R: Concept of vulnerable/unstable plaque. Arterioscler Thromb Vasc Biol 30: 1282-1292, 2010.

5. Didangelos A, Simper D, Monaco C and Mayr M: Proteomics of acute coronary syndromes. Curr Atheroscler Rep 11: 188-195, 2009.

6. Libby P, Ridker PM and Hansson GK: Progress and challenges in translating the biology of atherosclerosis. Nature 473: 317-325, 2011.

7. Moore KJ and Tabas I: Macrophages in the pathogenesis of atherosclerosis. Cell 145: 341-355, 2011.

8. Wang P, Wu T, Zhou H, Jin Q, He G, Yu H, Xuan L, Wang X, Tian L, Sun Y, et al: Long noncoding RNA NEAT1 promotes laryngeal squamous cell cancer through regulating miR-107/CDK6 pathway. J Exp Clin Cancer Res 35: 22, 2016.

9. Cooper DR, Carter G, Li P, Patel R, Watson JE and Patel NA Long non-coding RNA NEAT1 associates with SRp40 to temporally regulate PPAR $\gamma 2$ splicing during adipogenesis in 3T3-L1 Cells. Genes (Basel) 5: 1050-1063, 2014.

10. Ke H, Zhao L, Feng X, Xu H, Zou L, Yang Q, Su X, Peng L and Jiao B: NEAT1 is required for survival of breast cancer cells through FUS and miR-548. Gene Regul Syst Bio 10 (Suppl 1): S11-S17, 2016
11. Mang Y, Li L, Ran J, Zhang S, Liu J, Li L, Chen Y, Liu J, Gao Y and Ren G: Long noncoding RNA NEAT1 promotes cell proliferation and invasion by regulating hnRNP A2 expression in hepatocellular carcinoma cells. Onco Targets Ther 10: 1003-1016, 2017.

12. Gao C, Zhang J, Wang Q and Ren C: Overexpression of lncRNA NEAT1 mitigates multidrug resistance by inhibiting ABCG2 in leukemia. Oncol Lett 12: 1051-1057, 2016.

13. Chai Y, Liu J, Zhang Z and Liu L: HuR-regulated lncRNA NEAT1 stability in tumorigenesis and progression of ovarian cancer. Cancer Med 5: 1588-1598, 2016.

14. Naganuma T, Nakagawa S, Tanigawa A, Sasaki YF, Goshima N and Hirose T: Alternative 3'-end processing of long noncoding RNA initiates construction of nuclear paraspeckles. EMBO J 31: 4020-4034, 2012.

15. Imamura K,Imamachi N, AkizukiG,Kumakura M,Kawaguchi A, Nagata K, Kato A, Kawaguchi Y, Sato H, Yoneda M, et al: Long noncoding RNA NEAT1-dependent SFPQ relocation from promoter region to paraspeckle mediates IL8 expression upon immune stimuli. Mol Cell 53: 393-406, 2014

16. Fox AH, Lam YW, Leung AK, Lyon CE, Andersen J, Mann M and Lamond AI: Paraspeckles: A novel nuclear domain. Curr Biol 12: 13-25, 2002.

17. Anantharaman A, Jadaliha M, Tripathi V, Nakagawa S, Hirose T, Jantsch MF, Prasanth SG and Prasanth KV: Paraspeckles modulate the intranuclear distribution of paraspeckle-associated $\mathrm{Ctn}$ RNA. Sci Rep 6: 34043, 2016. International (CC BY-NC-ND 4.0) License. 